\title{
FLoRACIÓN DE ESPECIES CON POTENCIAL APÍCOLA EN EL BOSQUE Nativo Formoseño, Distrito Chaqueño Oriental (Argentina)
}

\author{
MIRTA CABRERA ${ }^{1}$, ANA ANDRADA² y LILIANA GALLEZ²
}

\begin{abstract}
Summary: Blooming of species with beekeeping potential in the native forest of Formosa, East Chaco District (Argentina). In order to do an inventory and a flowering calendar based on the plant species available in the native forest of the East of Formosa, the vegetation surveys were carried out in areas of $2 \mathrm{Km}^{2}$ surrounding 14 apiaries. From the 2009 through the 2012 beekeeping seasons, 198 species of Angiospermae were identified and their blooming was registered. Of these species, $94.5 \%$ corresponded to the native flora. Fabaceae, Asteraceae, Solanaceae, Sapindaceae and Polygonaceae were the richest plant families in taxa. The flowering periods lasted from mid August to early April. The maximum flowering supplies were recorded between October and December. Woody species dominated over herbaceous species mainly during the spring, within the three studied seasons. The aquatic and wetlands vegetation was characterized by its long-lasting flowering during the three-year study period. Abundant blooming of the species that integrate the native forest of Formosa is a valuable resource for beekeeping.
\end{abstract}

Key words: Floristic resources, flowering calendar, Formosa.

\begin{abstract}
Resumen: Con el propósito de inventariar y conocer el ritmo de floración de las especies vegetales de interés apícola, disponibles en la región del este del bosque nativo formoseño, se eligieron 14 apiarios y en cada uno de ellos se definió un área donde se realizaron los relevamientos de la vegetación. Durante las temporadas apícolas 2009-2012 se registró la floración de un total de 198 Angiospermae, de las cuales el $94,5 \%$ correspondieron a la flora nativa. Las familias con mayor diversidad de especies fueron Fabaceae, Asteraceae, Solanaceae, Sapindaceae y Polygonaceae. El período de floración se extendió desde mediados de agosto hasta principios de abril. La oferta máxima de floración se registró entre octubre y diciembre. En las tres estaciones de estudio se observó predominio de las especies leñosas $(67 \%)$ sobre las herbáceas, especialmente en primavera. La vegetación acuática y de humedales se caracterizó por su prolongada floración. La abundante floración del conjunto de especies que integran el bosque nativo formoseño constituye un valioso recurso de interés para la apicultura.
\end{abstract}

Palabras claves: Recursos florísticos, calendario de floración, Formosa.

\section{INTRODUCCIÓN}

Los inventarios y estudios de floración de las especies vegetales que componen un hábitat son esenciales para conocer los recursos apibotánicos disponibles en una región. Esta información es una herramienta útil para la conservación de la flora nativa y su posible beneficio en la actividad forestal, ornamental, industrial, turística, etc. En las

\footnotetext{
1 Facultad de Humanidades, Universidad Nacional de Formosa. Av. Gutnisky, (3200) Formosa.

2 Departamento de Agronomía, Universidad Nacional del Sur. Altos del Palihue, (8000) Bahía Blanca.
}

prácticas apícolas el conocimiento del calendario de floración es un instrumento valioso en el momento de planificar el aprovechamiento del potencial melífero.

En Argentina se han realizado estudios sobre calendarios de floración de especies de interés apícola en diferentes regiones fitogeográficas. Se destacan las publicaciones de Aramayo et al. (1993), Gurini \& Basilio (1995), Tellería (1993 y 1995), Lusardi et al. (2001) en la Provincia Pampeana; Fagundez (2011) en el área comprendida entre la provincia Pampeana y el Espinal; Andrada \& Gallez (2000), Andrada (2001 y 2003) en el Espinal; Forcone (2003), Naab \& Tamame (2007), Tamame (2011) en el Monte; Forcone \& Kutscher 
(2006) en la Provincia Subantártica y Forcone \& Muñoz (2009) en la Provincia Patagónica.

El estudio de los recursos apibotánicos de la Provincia Fitogeográfica Chaqueña ha sido abordado por diferentes investigadores (Salgado, 1999, 2006; Arbo et al., 2002; Nuñez Camelino, 2000; Cáceres \& Sánchez, 2004).

En Formosa la apicultura presenta un gran desarrollo en el sector oeste, mientras que en el este, aún es una actividad netamente familiar. El número estimado de apiarios en el territorio provincial asciende a 700 , con 22.000 colmenas y en el año 2010 se registró una producción de miel de 500.000 Kg (Ministerio de Producción de la Provincia de Formosa).

Este trabajo se enmarca en un proyecto integrado, cuyo propósito es valorizar las mieles del bosque nativo formoseño. El objetivo de este estudio fue inventariar y conocer el ritmo de floración de las especies vegetales disponibles en el área.

\section{Materiales y Métodos}

\section{Descripción del área}

El área de estudio se localiza al este de la provincia de Formosa, entre los $26^{\circ} 30^{\prime}$ y $25^{\circ}$ $05^{\prime} \mathrm{S}$ y $\operatorname{los} 57^{\circ} 39^{\prime}$ y $60^{\circ} \mathrm{O}$ (Fig.1); ocupa los departamentos Laíshi, Pilcomayo, Pilagás, Pirané, Formosa y el este del Departamento Patiño. Desde el punto de vista fitogeográfico, está comprendida en el Distrito Chaqueño Oriental (Cabrera, 1994), también denominado Parque Chaqueño Oriental (Ragonese \& Castiglioni, 1970) o Chaco Húmedo (Burkart et al., 1999). El clima es subtropical cálido con estación seca, el promedio de la temperatura en verano es de $26^{\circ} \mathrm{C}$ y en invierno de $16^{\circ} \mathrm{C}$ y la pluviometría varía de $1250 \mathrm{~mm}$ al este a $900 \mathrm{~mm}$ al oeste. Tres importantes cursos de agua surcan la zona de estudio: los ríos Paraguay al este, Bermejo al sur y Pilcomayo al norte. El área se encuentra afectada por anegamientos periódicos producidos

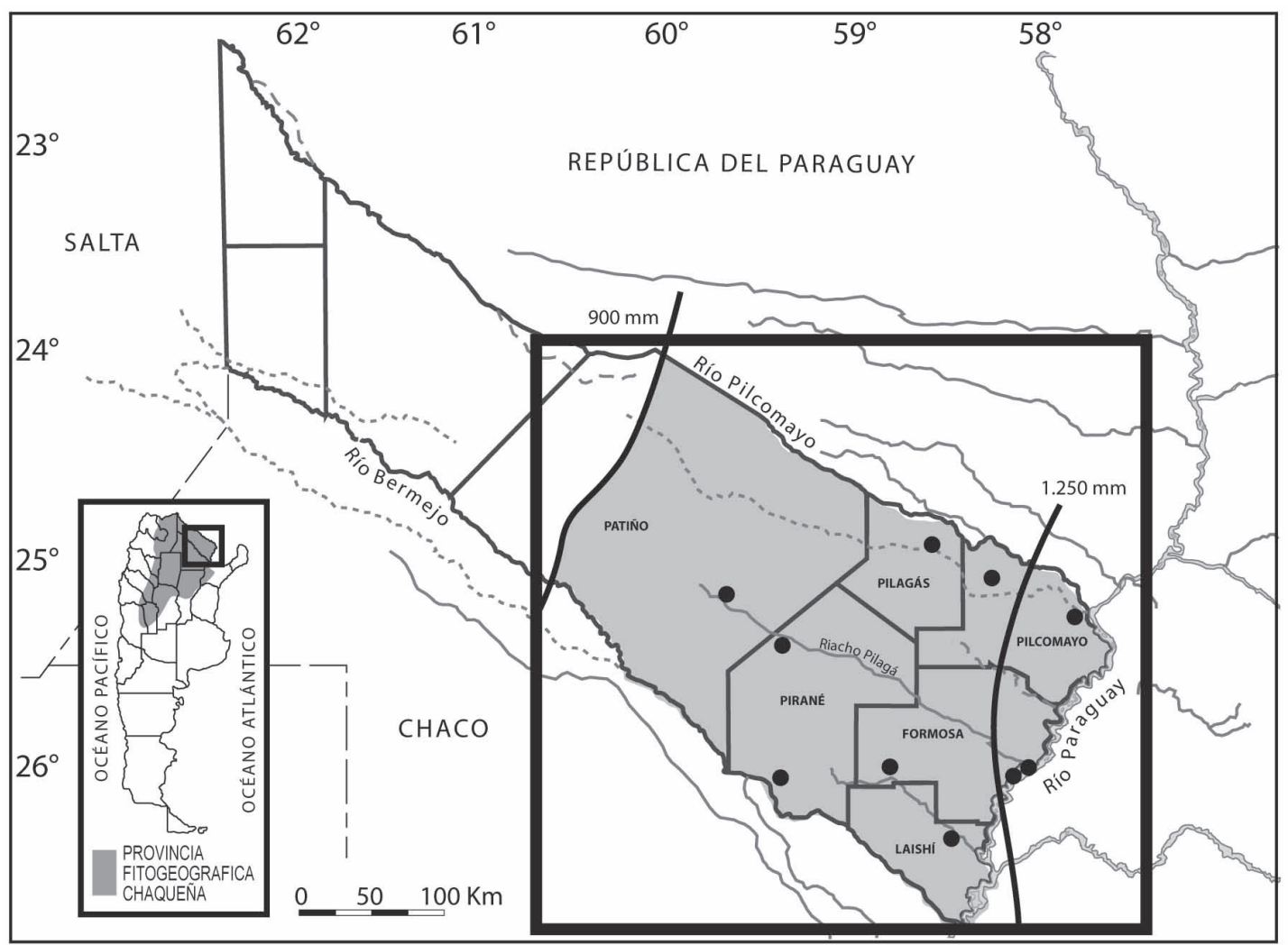

Fig.1.Ubicación geográfica del área de muestreo. Los puntos indican las localidades donde estaban asentados los apiarios. 


\section{Cabrera et al. - Floración de especies con potencial apícola en Bosque Nativo}

por lluvias y desbordes fluviales que establecen un paisaje heterogéneo. Se distinguen diferentes unidades ambientales: bosques, pastizales, sabanas y bañados con una gran diversidad de especies vegetales (Maturo et al., 2005).

El tipo de vegetación predominante en el Parque Chaqueño Oriental es el bosque nativo, donde se distinguen Schinopsis balansae, Aspidosperma quebracho blanco, Gleditsia amorphoides, Prosopis nigra var. nigra, Ziziphus mistol, Caesalpinia paraguariensis, Geoffroea decorticans, entre otros. En los suelos bajos e inundables se visualizan los palmares de Copernicia alba. A lo largo de ríos, lagunas y esteros se destaca la presencia de Tessaria integrifolia, Salix humboldtiana y especies hidrófitas como Cyperus giganteus Vahl.; y los camalotales flotantes de Eichhornia crassipes, Eichhornia azurea (Sw.) Kunth acompañados por Sagittaria montevidensis (Cabrera, 1994). Las actividades dominantes son la ganadería y la explotación forestal, en tanto que la agricultura ocupa una pequeña proporción de la región.

\section{Estudios florísticos}

Durante las temporadas apícolas primaveral y estival comprendidas entre los años 20092012, se registró la fenología de floración de las Angiospermas presentes en inmediaciones de 14 apiarios. En cada uno de ellos se definió un área de $2 \mathrm{Km}$ de radio donde se realizaron los relevamientos de la vegetación según el criterio de Anderson \& Hubritch (1940) indicando "inicio de floración", "floración plena" y "fin de floración-comienzo de fructificación”. Se observó un mínimo de 10 ejemplares de cada especie, con una frecuencia quincenal, y se registraron las tres fenofases antes mencionadas.

Se estimó la abundancia - cobertura de la vegetación de acuerdo con la escala de Braun Blanquet (1979). La información se agrupó en tres zonas, considerando la homogeneidad de la composición florística. Las zonas norte $(\mathrm{N})$ y sur $(\mathrm{S})$ están separadas por el Riacho Pilagá y limitadas al oeste por el Departamento de Patiño. La zona oeste (O) comprende la mayor parte del Departamento de Patiño y está limitada al oeste por la isoyeta de $900 \mathrm{~mm}$ (Fig. 1). Si bien en esta última zona el relevamiento se realizó en una única localidad, se decidió reportarla por separado debido a sus características agroecológicas. Este departamento ha sido diferenciado de los lindantes, en un estudio de zonificación agro-económica (SAGPyA, 2004).

Se coleccionaron e identificaron ejemplares botánicos utilizando los estudios florísticos de la región (Burkart, 1979, 1974; Burkart et al., 1987; Burkart \& Bacigalupo, 2005) y los taxa fueron validados (Instituto de Botánica Darwinion; Tropicos; International Plant Names Index). Los ejemplares fueron depositados en el herbario del IBONE-CONICET.

Los taxa pertenecientes a las familias Poaceae y Cyperaceae no fueron relevados por el escaso valor apícola que presentan (Tabla 1).

\section{Resultados y Discusión}

Durante el período de estudio se registró la floración de 198 taxa, de las cuales el 94,5\% pertenecen a la flora autóctona de Sudamérica. Las especies identificadas pertenecen a 60 familias de las Angiospermas, destacándose Fabaceae, Asteraceae y Solanaceae por su mayor diversidad $(33,26$ y 15 especies respectivamente); otras familias bien representadas fueron Sapindaceae y Polygonaceae (Tabla 1).

La oferta floral de especies nativas y exóticas se extiende por un largo período que comienza a principios de agosto y presenta su máxima expresión en diciembre. A partir de enero se reduce sensiblemente el número de especies en flor, tanto nativas como exóticas (Fig. 2).

La primera quincena de agosto se observó una abundante disponibilidad de especies leñosas, entre las que se registraron las Fabáceas Acacia aroma, A. caven var. caven, A. praecox y Geoffroea decorticans, acompañadas por Allophylus edulis, Salix humboldtiana y Vassobia breviflora.

En la primavera irrumpieron los "algarrobales" compuestos por Prosopis alba var. alba, P. nigra, P. affinis, P. hassleri var. hassleri, P. kuntzei, P. ruscifolia, y junto a ellos florecieron Eugenia uniflora, Sapium haematospermum, Parkinsonia aculeata y Ziziphus mistol. Durante éste período se encontraron en flor las hierbas nativas Acicarpha tribuloides, Phyla nodiflora var. reptans, Vicia macrograminea, Heliotropium elongatum y Senecio grisebachii.

Al final de la primavera y comienzo del verano las Anacardiáceas Astronium balansae, Schinopsis 


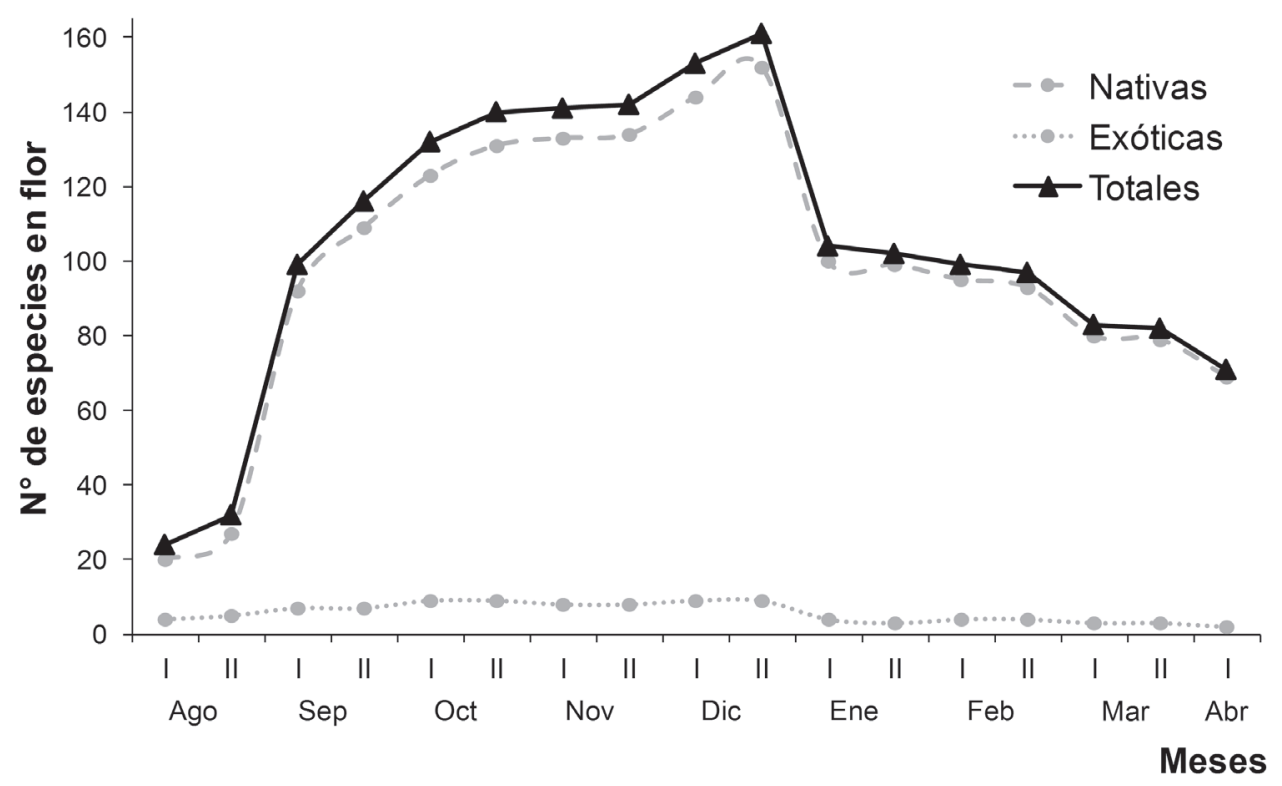

Fig. 2. Oferta de Floración en el Distrito Chaqueño Oriental (Formosa). Se representa el número de especies totales, nativas y exóticas. I y II, indican la primera y segunda quincena de cada mes.

balansae y Schinus fasciculatus var. fasciculatus, iniciaron su prolongada floración. Durante el período mencionado se observaron en flor en los márgenes del bosque algunas Verbenáceas arbustivas: Aloysia virgata var. platyphylla y Aloysia gratissima var. chacoensis. En los humedales predominó Copernicia $a l b a$, especie indicadora de suelos bajos, que crece en parcelas casi puras principalmente en las zonas N y S.

La floración de las lianas y enredaderas Ipomoea cairica, Dolichandra unguis-cati, Araujia odorata, Passiflora caerulea, Clematis montevidensis var. montevidensis, Cardiospermum halicacabum y Paullinia pinnata se superpuso, a lo largo de todo el período, con la de la vegetación leñosa presente en los diferentes estratos del bosque (Tabla 1).

Durante el verano, se destacaron las Asteráceas arboreo-arbustivas y herbáceas denominadas vulgarmente "chilcas": Baccharis salicifolia, B. punctulata, Eupatorium inulaefolium, E. ivifolium y Tessaria integrifolia acompañadas por Solidago chilensis var. megapotamica.

En las tres estaciones de estudio se observó predominio de las especies leñosas (67\%) sobre las herbáceas, especialmente en primavera (Fig. 3). La comunidad vegetal de esteros, lagunas y bañados se caracterizó por su prolongada floración. En estos ambientes se identificaron los hidrófitos emergentes Sagittaria montevidensis ssp. montevidensis, S. guayanensis, Pontederia cordata var. cordata, Echinodorus grandiflorus, Ludwigia sp, Polygonum hydropiperoides var. setaceum y P. punctatum y las plantas flotantes Eichhornia crassipes, Nymphoides indica y Hydrocleys nymphoides (Tabla 1).

En el Parque Chaqueño Oriental, el período de floración se extendió desde agosto hasta abril, con una oferta máxima entre los meses de octubre y diciembre (Fig. 2). Este intervalo es comparable con el observado en la provincia del Chaco (Salgado, 2006) y en la región Pampeana (Tellería, 1993). Mientras, que en las Provincias Patagónica y del Monte el calendario de floración es más corto, se extiende de septiembre a marzo (Forcone, 2003; Forcone \& Muñoz, 2009) y en el Delta del Paraná abarca prácticamente todo el año (Gurini \& Basilio, 1995).

Algunas especies de la familia Fabaceae y $S$. balansae presentaron, por fuera del período principal, floraciones sucesivas de menor intensidad, breves y en ocasiones con producción de frutos (Tabla 1). Estas observaciones coinciden con estudios realizados en el distrito del Caldén (Andrada \& Tellería, 2005) y en las provincias del Chaco (Salgado, 2006) y Formosa (Cáceres \& Sánchez, 2004). 
M. Cabrera et al. - Floración de especies con potencial apícola en Bosque Nativo

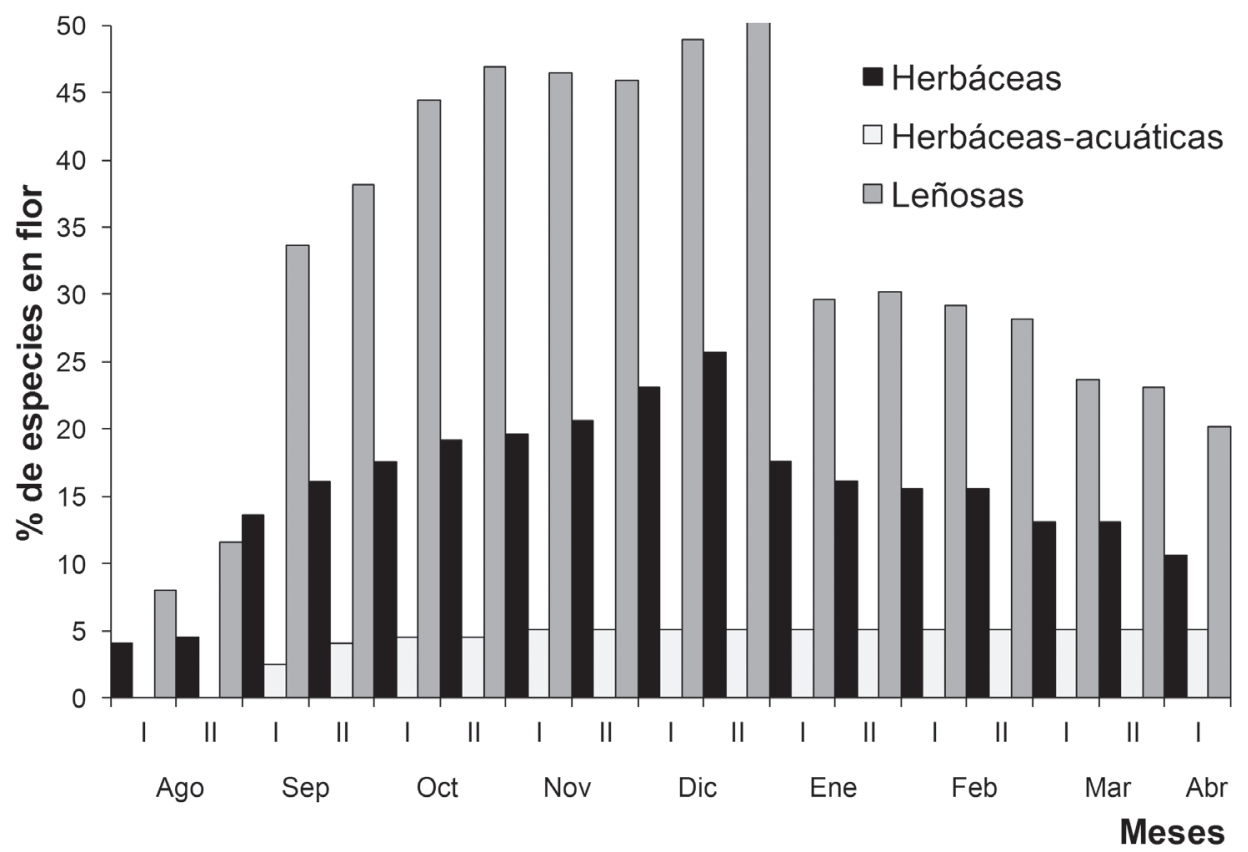

Fig. 3. Floración de especies herbáceas, herbáceas-acuáticas y leñosas. I y II, indican la primera y segunda quincena de cada mes.

Tabla 1. Períodos de floración de 198 especies registradas en el Distrito Chaqueño Oriental (Formosa) (2009-2012). (*) Especies exóticas. H: hábito: h: hierba, a: árbol, ar: arbusto, aa: arbóreo-arbustivo, hp: hierba palustre, sa: subarbusto, e: enredadera, I: liana, ha: hierba acuática, p: palmera, sa-p: subarbusto parásito, h-sa: hierba o subarbusto, h-e: hierba o enredadera, h-ar: hierba o arbusto. Trazo grueso:

floración plena. Trazo punteado: inicio y fin de floración. I y II: primera y segunda quincena de cada mes. Ab-Cob.: Abundancia-cobertura de los individuos: + (raros); 1 (escasos); 2 (numerosos), 3 y 4: (muy numerosos). Zonas: $\mathrm{N}$ (norte), S (sur) y O (oeste).

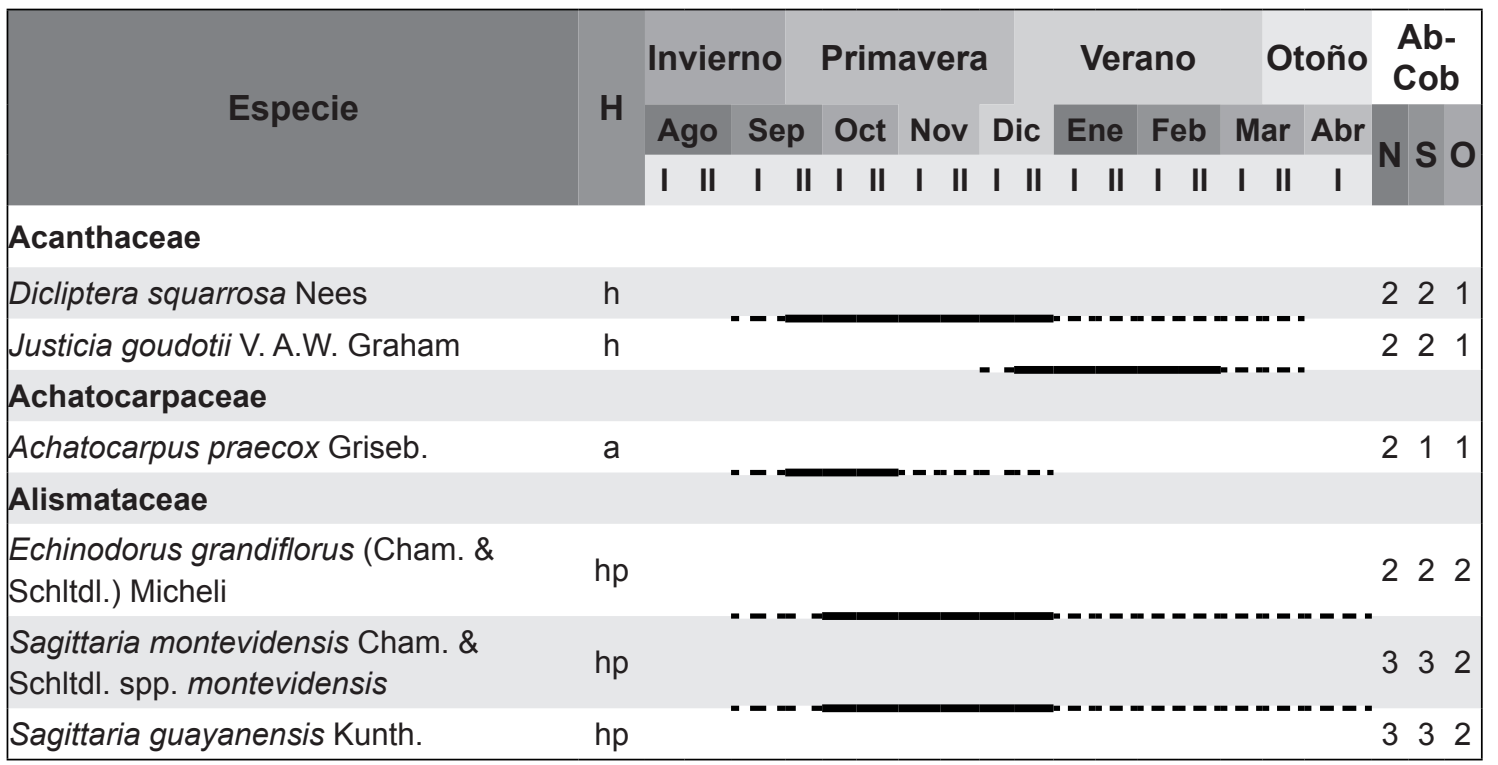


Bol. Soc. Argent. Bot. 48 (3-4) 2013

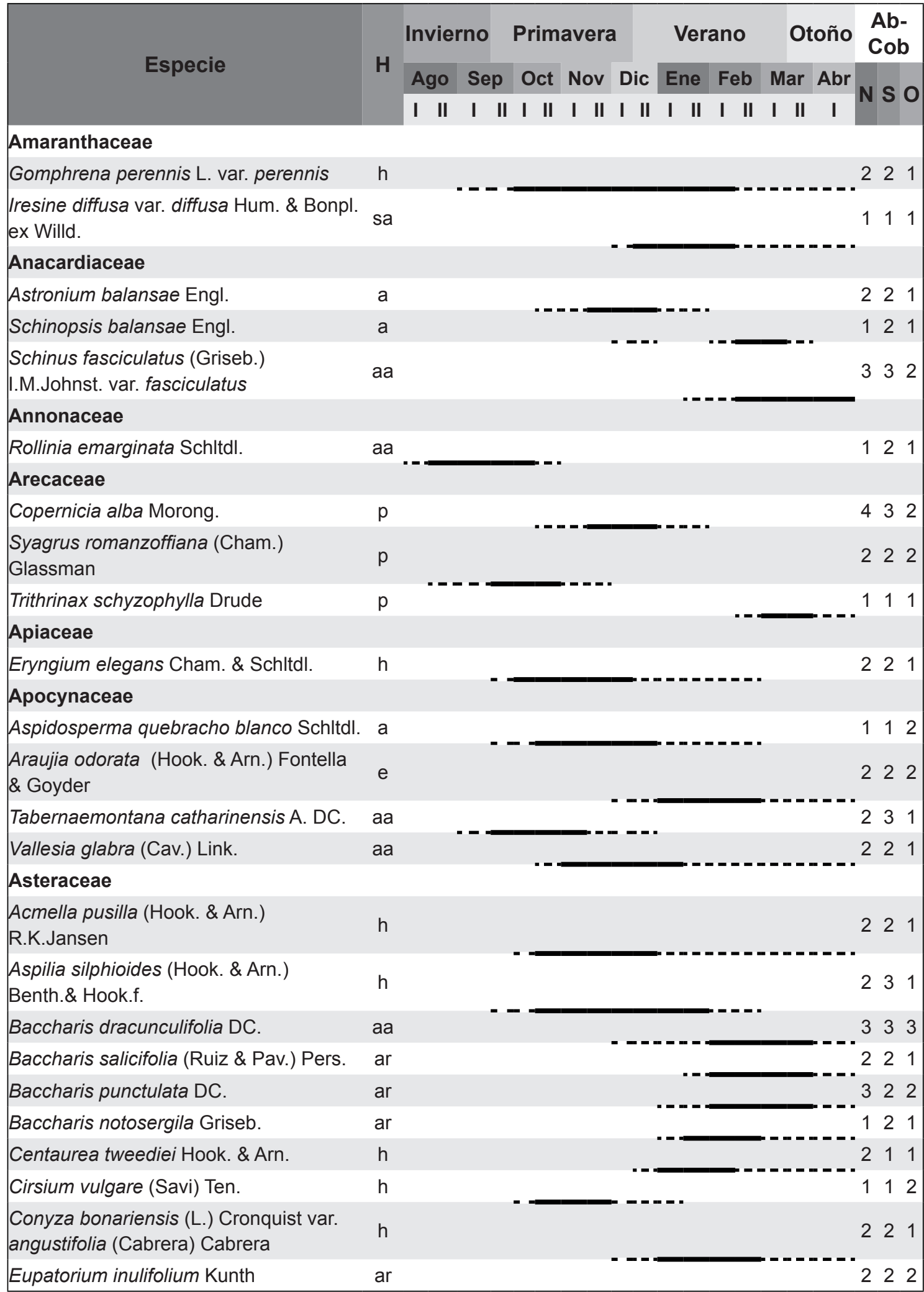


M. Cabrera et al. - Floración de especies con potencial apícola en Bosque Nativo

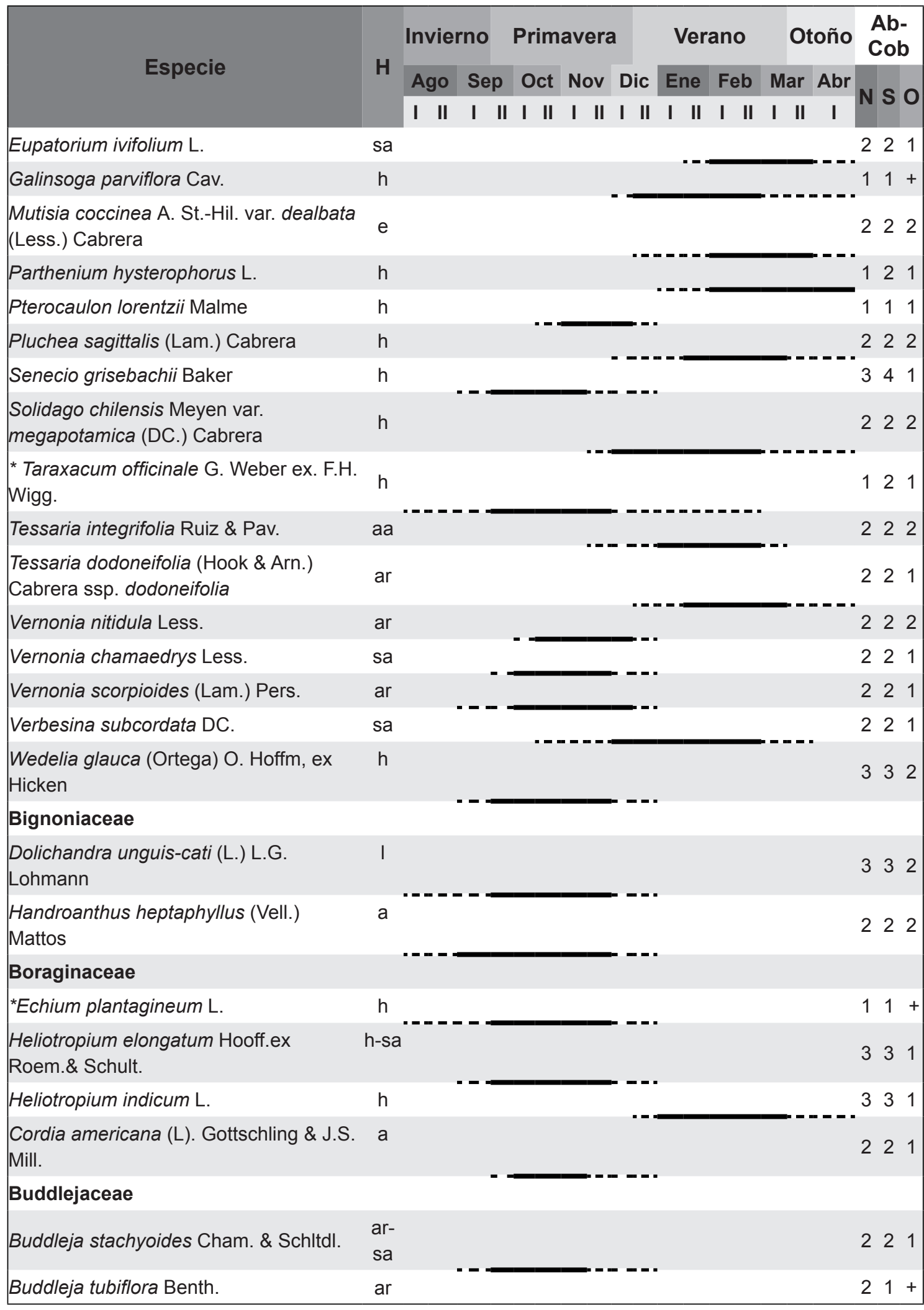


Bol. Soc. Argent. Bot. 48 (3-4) 2013

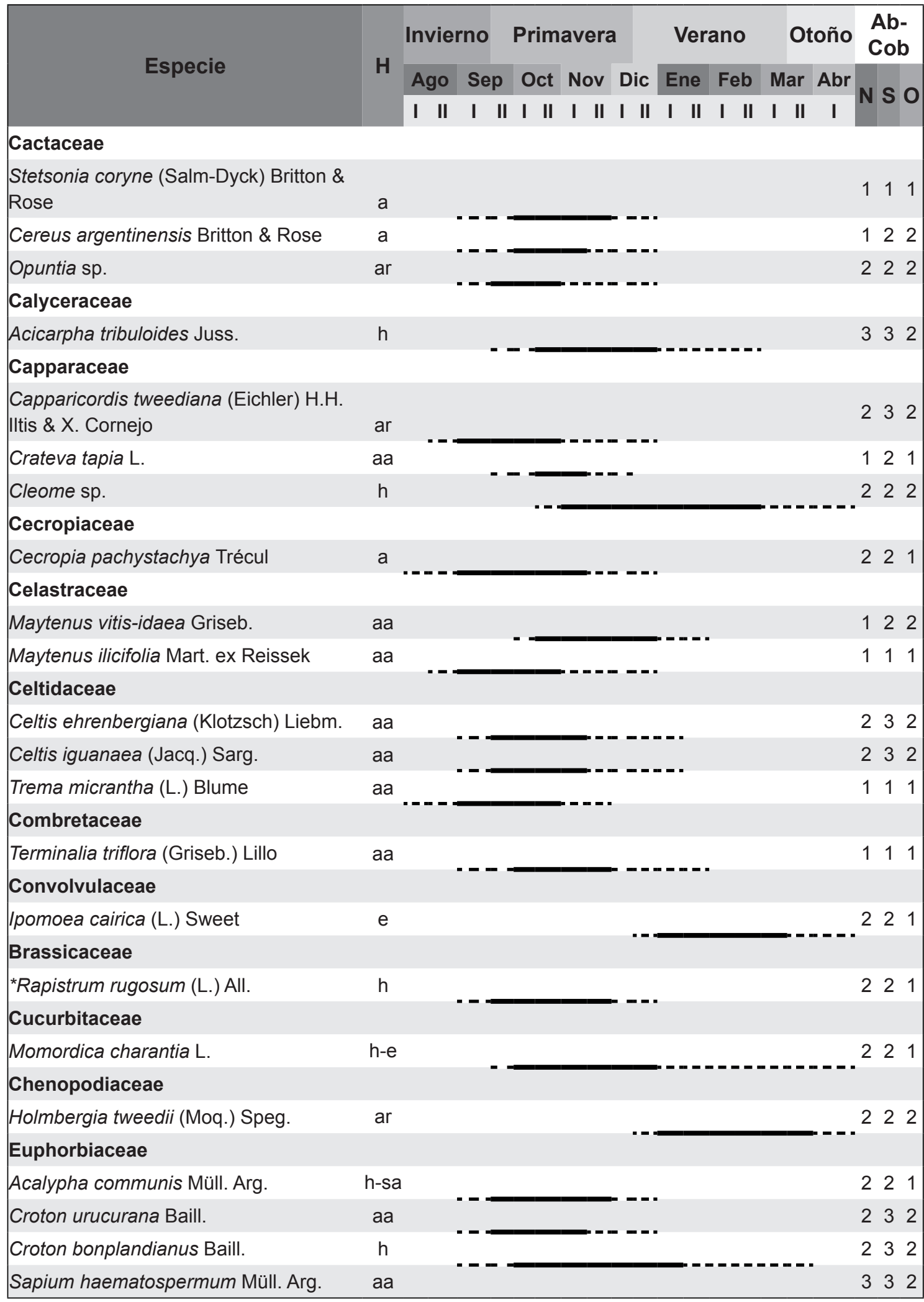




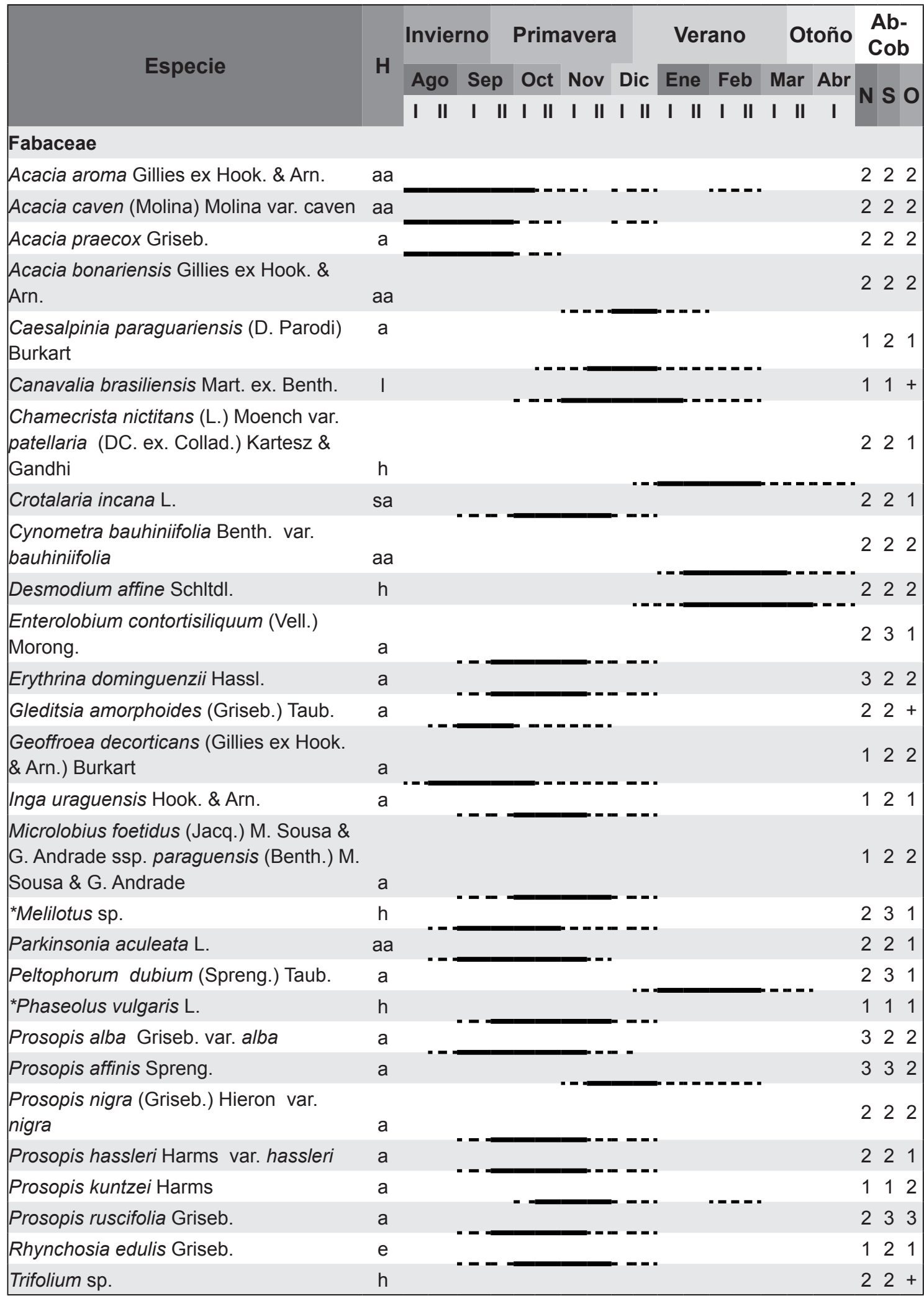


Bol. Soc. Argent. Bot. 48 (3-4) 2013

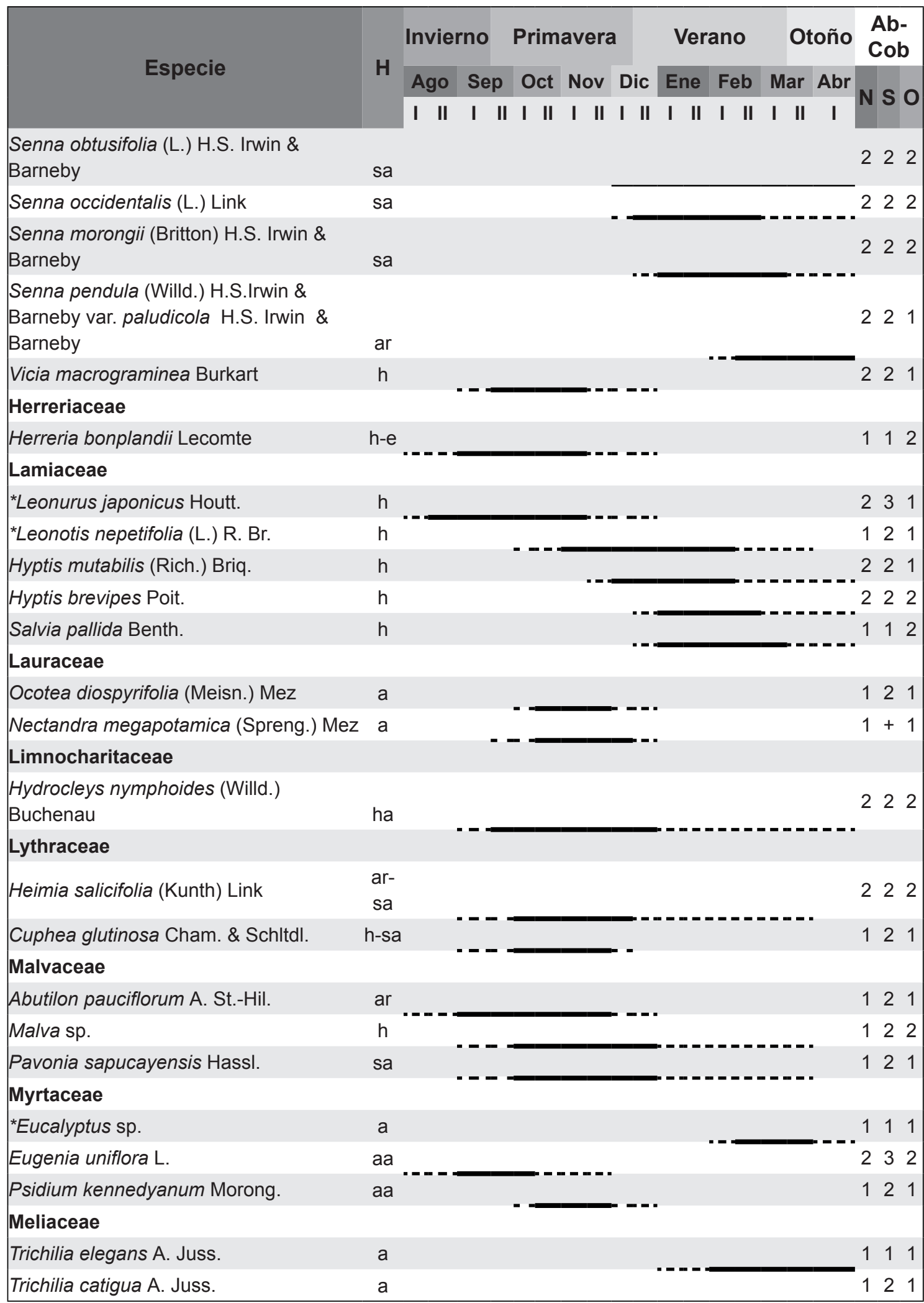


M. Cabrera et al. - Floración de especies con potencial apícola en Bosque Nativo

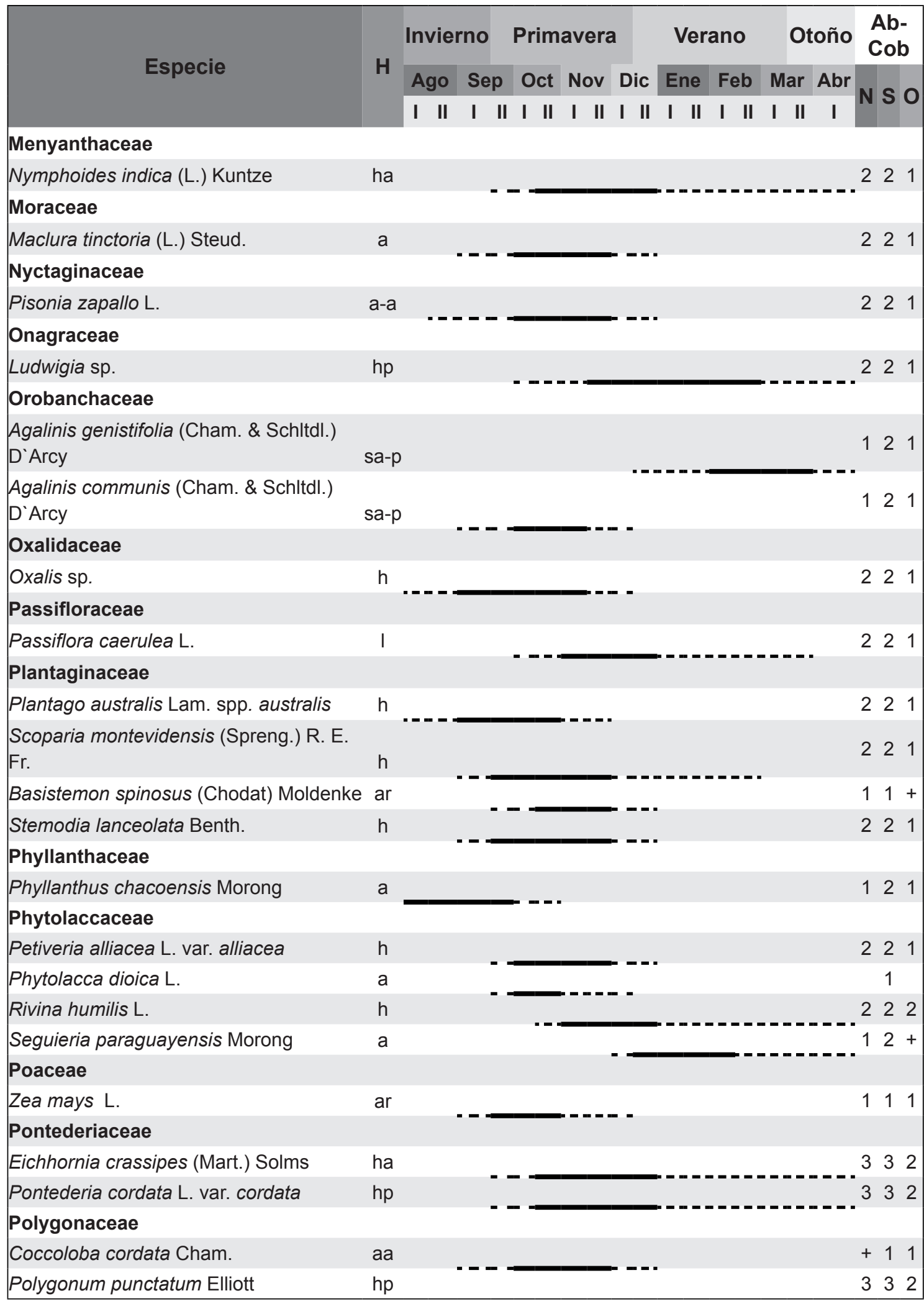


Bol. Soc. Argent. Bot. 48 (3-4) 2013

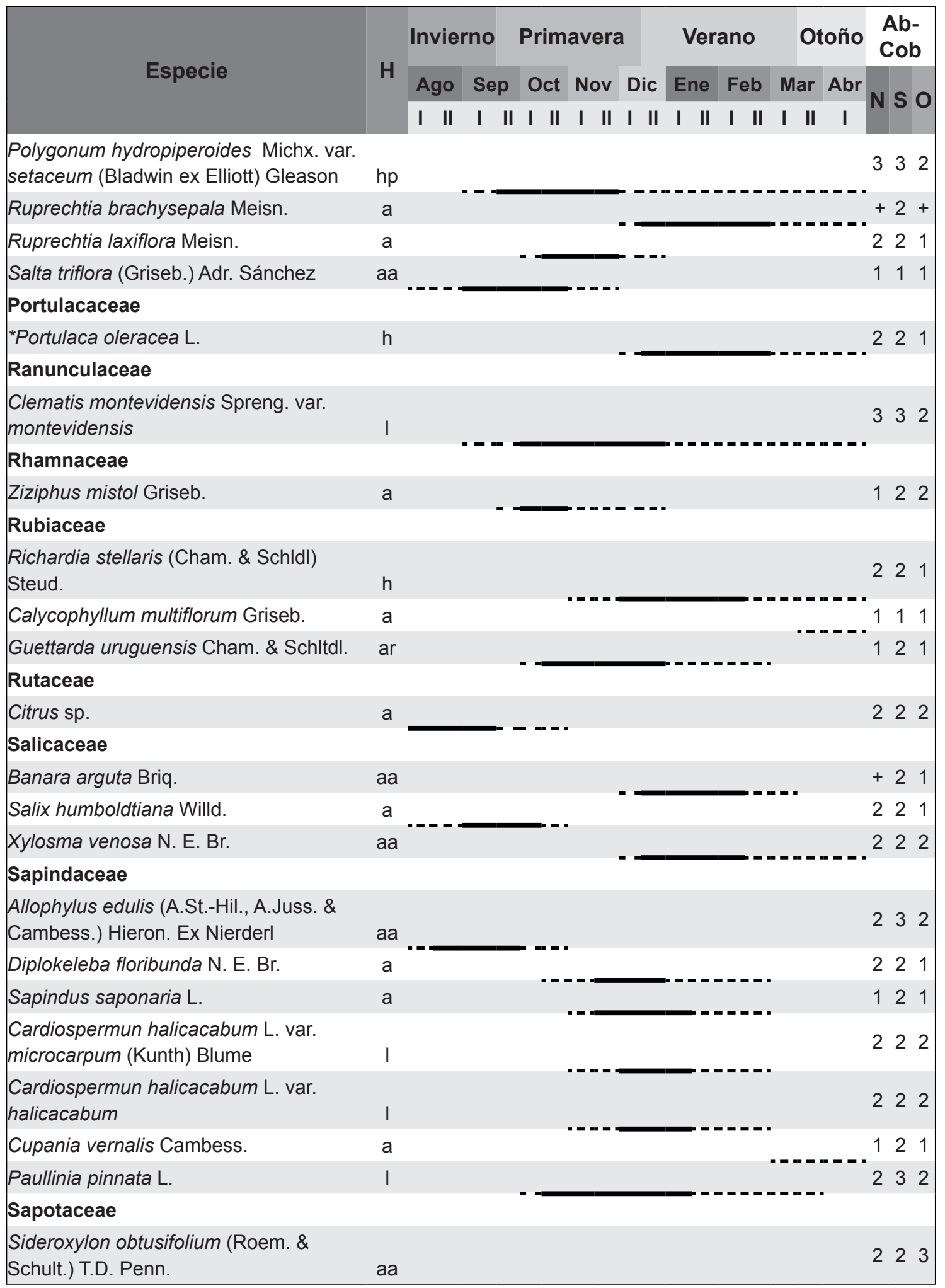


M. Cabrera et al. - Floración de especies con potencial apícola en Bosque Nativo

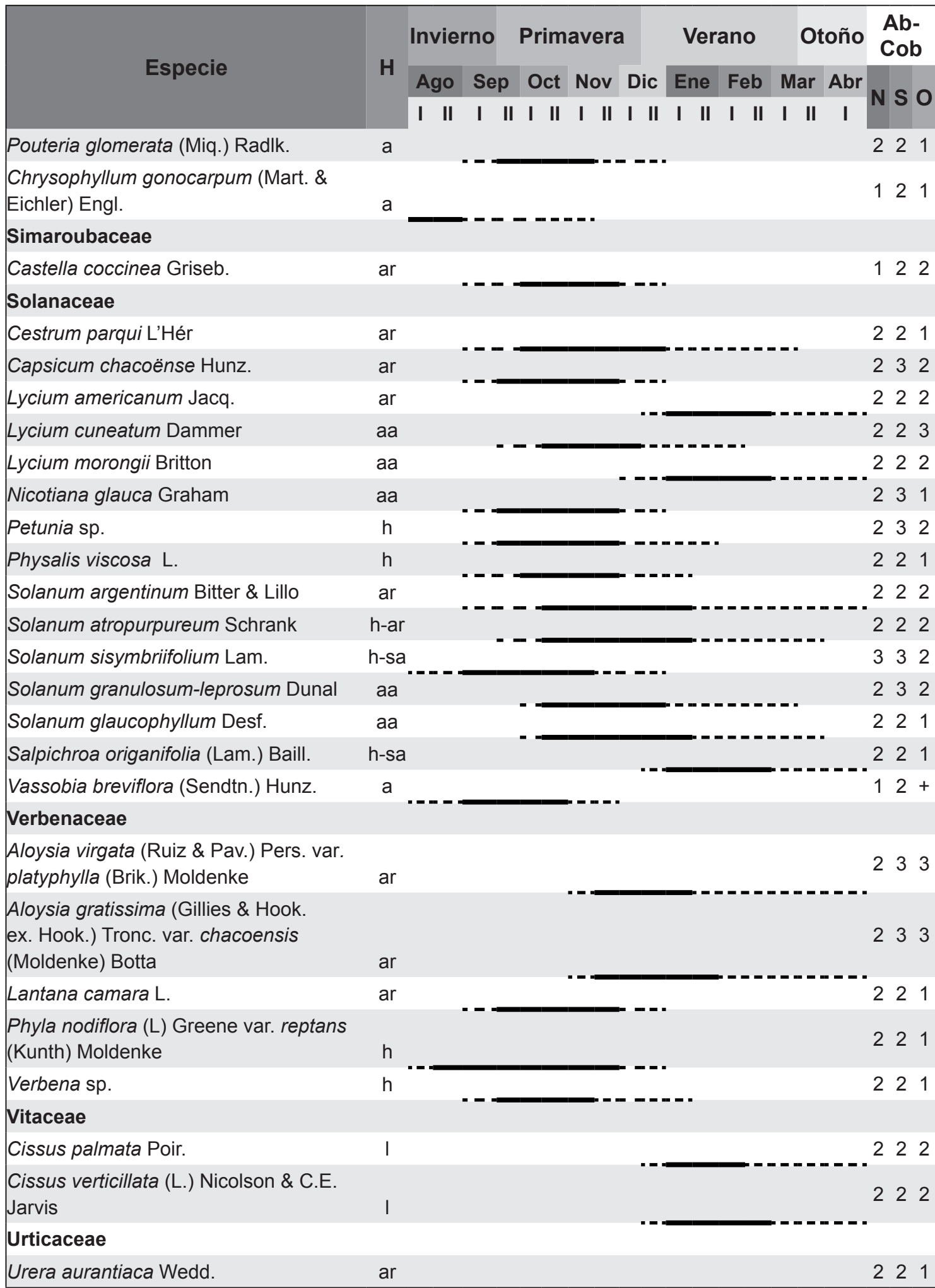


Más del la mitad de las especies presentadas en la Tabla 1 han sido mencionadas en estudios melisopalinológicos de la Provincia Chaqueña (Basilio \& Noetinger, 2000; Jiménez et al., 2004; Salgado, 2006; Cabrera, 2006; Cabrera \& Andrada, 2009 y Cabrera et. al., 2011). El grado de solapamiento de los taxa cuya floración se estudió en este trabajo con el polen presente en las mieles concuerda con la información presentada por Andrada (2001) en el sur del distrito del Caldén. La dominancia de pólenes de especies arbóreas en numerosas mieles analizadas en los trabajos antes mencionados, como Bulnesia sarmientoi Lorentz ex. Griseb., Geoffroea decorticans, Copernicia alba, Eugenia uniflora, Prosopis alba, Schinopsis balansae, Schinus spp. y Ziziphus mistol, corroboran la importancia de la vegetación arbórea como recurso nectarífero.

\section{Conclusiones}

En el este formoseño, la flora con potencial apícola presenta una gran diversidad de especies y está constituida casi exclusivamente por vegetación autóctona.

El período de floración se extendió desde agosto hasta abril cubriendo, de acuerdo a la información bibliográfica, el período de actividad de las colmenas en la región.

Entre los taxa de valor apícola censados en las cercanías de los apiarios dominaron en número las especies leñosas por sobre las herbáceas.

La vegetación acuática y de humedales mostró un extenso intervalo de floración y por ello representa un buen aporte melífero en la región.

La abundante y extensa floración del conjunto de especies que integran el bosque nativo formoseño constituye un valioso recurso de interés para la apicultura.

\section{Agradecimientos}

A los productores de la zona por su hospitalidad y buena predisposición para facilitarnos el acceso a los predios. A Gelina Esther Pieszko y Darvin Cáceres por su colaboración en la determinación de las plantas. A Cristina Salgado por su permanente colaboración y acompañamiento. A la Secretaría de Ciencia y Técnica de la Universidad Nacional de Formosa por suministrar el financiamiento del proyecto.

\section{Bibliografía}

ANDERSON, E. \& L. HUBRITCH. 1940. A methods for describing and comparing blooming season. Bull. Torrey Club 67: 639-649.

ANDRADA, A. \& L. GALLEZ. 2000. Flora apícola del Caldenal. Actas de resúmenes de las IV Jornadas Bienales de Apicultura, Fortin Mercedes- Pedro Luro, Buenos Aires.

ANDRADA, A. 2001. Estudio de la flora melífera y polinífera en la zona sur del Distrito del Caldén, Provincia del Espinal. Tesis doctoral. Universidad Nacional del sur.

ANDRADA, A. 2003. Flora utilizada por Apis mellifera L. en el sur del Caldenal (Provincia Fitogeográfica del Espinal). Revista Mus. Argent. Ci. Nat. Bernardino Rivadavia 5: 329-336.

ANDRADA, A. \& TELleRÍA, M. C. 2005. Pollen collected by honey bees (Apis mellifera L.) from south of Caldén distrito (Argentina): botanical origin and protein content Grana 44: 1-8.

ARAMAYO, E., A. VALLE, A. ANDRADA \& S. LAMBERTO. 1993. Calendario de floración de árboles y especies espontáneas frecuentes en Bahía Blanca. Parodiana 8: 265-270.

ARBO, M. M., M. LOPEZ, A. SCHININI \& G. PIESZKO. 2002. Plantas palustres del Macrosistema Iberá. Actas de Comunicaciones Cientificas y Tecnológicas de la UNNE [online]. Disponible en: http://www. unne.edu.ar [Acceso: 10 de marzo de 2011].

BASILIO, A. \& M. NOETINGER. 2000. Análisis polínico de mieles de la Región Chaqueña: comparación del origen floral entre las zonas Domo Central y Esteros, Cañadas y Selvas de Rivera. INTA 31: 127-134.

BRAUN-BLANQUET, J. 1979. Fitosociología. Bases para el estudio de las comunidades vegetales. Ediciones H. Blume, Madrid.

BURKART, A. (Ed.) 1974. Flora Ilustrada de Entre Ríos. Parte VI: Dicotiledóneas Metaclamídeas B: Rubiales, Cucurbitales, Campanulales. Colección Cientifica del INTA 6: 1-554.

BURKART, A. (Ed.) 1979. Flora Ilustrada de Entre Ríos. Parte V: Dicotiledóneas Metaclamídeas A: Primulales a Plantaginales. Colección Científica del INTA 6: 1-606.

BURKART, R., N. O. BÁRBARO, R. O. SÁNCHEZ \& D. A. GÓMEZ. 1999. Eco-regiones de la Argentina. Administración de Parques Nacionales, Programa Desarrollo Institucional Ambiental, Buenos Aires.

BURKART, A., N.S. TRONCOSO \& N. BACIGALUPO. 1987. Flora Ilustrada de Entre Ríos Parte III: 


\section{Cabrera et al. - Floración de especies con potencial apícola en Bosque Nativo}

Dicotiledóneas Arquiclamídeas A: Salicales a Rosales. Colección Científica del INTA 6: 1-763.

BURKART, A. \& N. BACIGALUPO. 2005. Flora Ilustrada de Entre Ríos. Parte IV. Colección Científica del INTA 6: 1-627.

CABRERA, A. 1994. Enciclopedia Argentina de Agricultura y Jardinería. 1st ed. T.2, Fasc. 1: Fitogeografía de la República Argentina. Editorial Acme S.A.C.I., Buenos Aires.

CABRERA, M. 2006. Caracterización Polínica de las Mieles de la Provincia de Formosa, Argentina. Revista Mus. Argent. Ci. Nat.Bernardino Rivadavia n.s. 8: 135-142.

CABRERA, M. \& A. ANDRADA. 2009. Origen floral de las mieles en la Región Húmeda del Distrito Chaqueño Oriental. Actas de resúmenes de las Jornadas de Ciencia y Tecnología 12: 148-150.

CABRERA, M., A. ANDRADA, L. GALLEZ \& D. IACONIS. 2011. Preliminary characterization of honeys from eastern Formosa Province, Argentina. Libro de resúmenes del $42^{\circ}$ Congreso Internacional de Apicultura, APIMONDIA 2011: 1-255.

CÁCERES, D. A. \& V. SÁNCHEZ. 2004. Fenología y Taxonomía de Especies Forestales de la Provincia de Formosa.1st de. Editorial Universitaria. Facultad de Recursos Naturales. Universidad Nacional de Formosa. Libro digital.

FAGÚNDEZ, G. 2011. Estudios de los recursos nectaríferos y poliníferos utilizados por Apis mellifera L. en diferentes ecosistemas del Departamento de Diamante (Entre Ríos, Argentina). Tesis doctoral. Universidad Nacional del Sur.

FORCONE, A. 2003. Floración y utilización de la flora apícola en el valle inferior del río Chubut (Patagonia Argentina). Bol. Soc. Argent. Bot. 38: 301-310.

FORCONE, A. \& A. KUTSCHKER. 2006. Floración de las especies de interés apícola en el noroeste de Chubut, Argentina. Rev. Mus. Argent. Cienc. Nat., n.s. 8: 151157.

FORCONE, A. \& M. MUÑOZ. 2009. Floración de las especies de interés apícola en el noroeste de Santa Cruz. Bol. Soc. Argent. Bot. 44: 393-403.

GURINI, L. \& A. BASILIO. 1995. Flora apícola en el Delta del Paraná. Darwiniana 33: 337-346.

INSTITUTO DE BOTÁNICA DARWINION. Flora del Conosur, Catálogo de Plantas Vasculares [online]. Disponible en http://www.darwin.edu.ar [Acceso: 8 de marzo de 2010].

INTERNATIONAL PLANT NAMES INDEX (IPNI). The Royal Botanic Gardens Kew The Harvard University Herbaria, and the Australian National Herbarium[online]. Disponible en http://www.ipni.org [Acceso: 15 de abril de 2010].

JIMENEZ, C., J. MAIDANA \& M. RODRIGUEZ. 2004. Origen floral y color de mieles de la Provincia de
Santiago del Estero, Argentina. Seminario Internacional de Calidad de miel. Rafaela, Santa Fé.

LUSARDI, M., A. SCANDIZZI, J. McCARGO, S. GATUSSO, L. ARDUSS \& C. CRISCI. 2001. Calendario de Floración de especies frecuentes en la ciudad de Rosario (Santa Fe, Argentina. Archivos de Alergia e Inmunología Clínica 32: 93-97.

MATURO, H., L. OAKLEY \& D. PRADO. 2005. Vegetación y posición fitogeográfica de la Reserva El Bagual. Temas de Naturaleza y ConservaciónMonografias de Aves Argentinas 4: 61-96.

NAAB, O. \& M. TAMAME. 2007. Flora apícola primaveral en la región del Monte de la provincia de La Pampa (Argentina). Bol. Soc. Argent. Bot. 42: 251-259.

NUÑEZ CAMELINO, A. 2000. Determinación apícola de las secciones $1^{\circ}, 2^{\circ}$ y $4^{\circ}$ del Departamento Saladas de la Provincia de Corrientes. En Actas de Comunicaciones Cientificas y Tecnológicas de la UNNE. www.unne. edu.ar.

RAGONESE, A. E. \& J. C. CASTIGLIONI. 1970. La vegetación del Parque Chaqueño. Bol. Soc. Argent. Bot. 11: $133-160$.

SAGPYA (Secretaría de Agricultura, Ganadería, Pesca y Alimentación), Ministerio de Economía de la República Argentina. 2004. Oficina de Riesgo Agropecuario (ORA) [online]. Disponible en: http:// www.sagpya.mecon.gov.ar [Acceso: 3/1/2005].

SALGADO, C. 1999. Comparación de los recursos apibotánicos en dos localidades del Dpto. Bella Vista. Actas de Comunicaciones Científicas y Tecnológicas de la UNNE, pp. 193-196.

SALGADO, C. 2006. Flora melífera en la provincia del Chaco. Ministerio de la Producción del Chaco. Editor PROSAP, Impreso por Infográfica, Resistencia (Argentina).

TAMAME, A. 2011. Estudio de la composición, disponibilidad y calidad de los recursos apícolas del Nordeste de la Pampa, provincia fitogeográfica del Monte, (República Argentina). Tesis doctoral. Universidad Nacional de la Plata.

TELLERIA, M.C. 1993. Floración et récolte du pollen par les abeilles domestiques (Apis mellifera L. var. ligustica) dans la pampa argentine. Apidologie 24: 109-120.

TELLERIA, M.C. 1995. Plantas de importancia apícola del Distrito Oriental de la Región Pampeana (Argentina). Bol. Soc. Argent. Bot. 30: 131-136.

TROPICOS. Missouri Botanical Garden [online]. Disponible en http://www.tropicos.org [Acceso: 15 de abril de 2010].

Recibido el 24 de julio de 2012, aceptado el 18 de febrero de 2013. 
